\title{
SUPPORING INFORMATION: HPLC-detector based on Hadamard-Transform Fluorescence Excitation-Emission-Matrix spectroscopy
}

\author{
Travis Ferguson ${ }^{1}$, Adam Bernicky ${ }^{1}$, Igor Kozin ${ }^{1}$, and Hans-Peter Loock ${ }^{1,2 *}$ \\ ${ }^{1}$ Department of Chemistry, Queen's University, Kingston, ON, Canada, K7L 4V1 \\ 2 Department of Chemistry, University of Victoria, Victoria, BC, Canada, V8W 2Y2
}

SUMMARY: in the supporting information we include computational details of the PARAFAC method, the determination of the misattribution of chromatographic peaks to the wrong constituents, and additional information on the calculation of the linear response range and limit of detection of the proposed technique.

\section{PARAFAC Analysis}

Parallel Factor Analysis (PARAFAC), also known generally as tensor rank decomposition, is a multidimensional decomposition technique which has been the model of choice for excitation-emission-matrix (EEM) data analysis for the past 20 years. ${ }^{1}$ A component in a PARAFAC model is defined by three component variables, i.e. its excitation loading, its emission loading, and its score. The component's excitation loading is a vector which is equivalent to the excitation spectrum of the fluorophore, while the emission loading is equivalent to the emission spectrum. It should be noted that since the excitation and emission loading vectors are independent of one another, using PARAFAC analysis to analyze an EEM dataset implies the validity of Kasha's rule. In cases where fluorophores do not follow Kasha's rule, PARAFAC is not the recommended method for modeling of the data. The third component variable is the component score which is proportional to the product of the quantum yield and concentration of the fluorophore. In most cases the quantum yield of a fluorophore is expected to remain constant, and then the concentration of the fluorophore can be calculated from the component's scores once a scaling factor is determined after performing a calibration.

PARAFAC uses a random initialization method. Each iteration uses a randomly determined initial set of values for each of the three component variables before minimizing the sum of squared residuals (SSR) between the model and the experimental data. The convergence criteria of this model are defined as the minimum difference between the SSR of one model and the following one, and can range from $10^{-2}$ to $10^{-20}$. The PARAFAC algorithm can sometimes converge at a local minimum on the SSR surface rather than the global minimum and multiple converged models (typically 10 to 20) were compared in our study to identify the model with the lowest SSR as the optimized model. Also, here, a constraint was used for PARAFAC models of EEM data to ensure the scores, excitation and emission loadings do not assume negative values.

\section{Misattribution and Identity of PARAFAC Components}

Since PARAFAC is a randomly initialized method and the components are not mutually exclusive, it is possible for two components to have similar or even identical emission or excitation loadings, but it is not possible for two components to share both. The misattribution seen in our "blind" PARAFAC models are primarily due to the similarity between the emission loadings of the two components (coumarin 440 and coumarin 450), and illustrate that PARAFAC had not been able to fully distinguish the two components. The addition of either higher resolution emission spectra or more excitation channels should help alleviate misattribution.

When analyzing EEM datasets it is possible to overfit a data set resulting in the attribution of two components to a single fluorophore. In our case we have a known number of fluorophores in our mixture (five) and the exact number of components can be used to model the data set. Indeed Figure S1 shows that all five components (Fig. S1d) have an associated coumarin dye that has a very similar EEM spectrum (Fig. S1c).

When comparing the raw EEM data collected from the chromatography of the solution containing all five dyes (Fig. $\mathrm{S} 1 \mathrm{~b})$ it is apparent that the coumarin and component spectra again are comparable.

Overfitting may sometimes be reduced by using higher spectral resolution or spectra with higher signal-to-noise ratios. In our study we deliberately selected components which have very similar emission or excitation spectra to provide a challenge to the detection system. Specifically, components 1,2, and 4 all have similar emission spectra and are all affected by misattribution seen in the PARAFAC models.

\section{Calculation of PARAFAC Misattribution}

The percentage of component misattribution was calculated by integrating the area of components known to elute at a given time $\left(P_{a}\right)$ and comparing that are to the area of peaks from components not expected at that point in time $\left(P_{i}\right)$. 
(a)

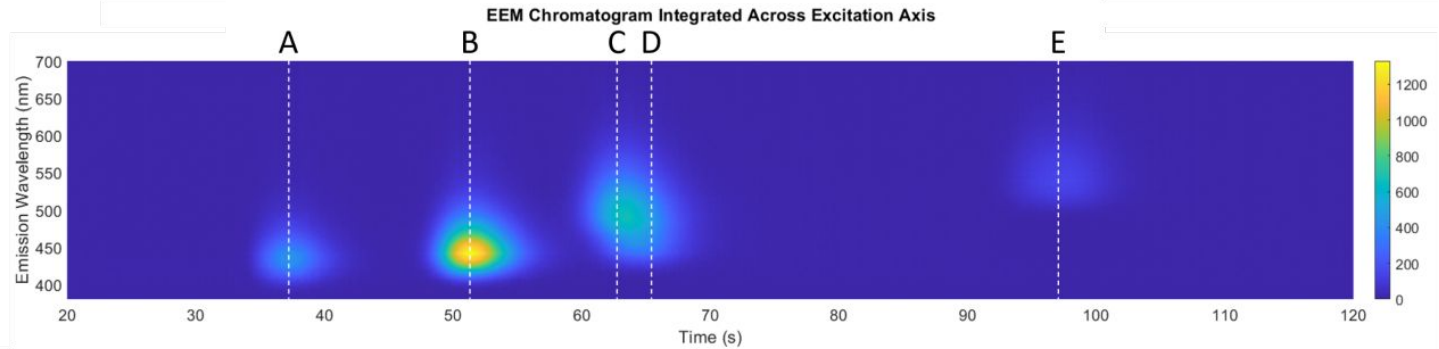

(b)
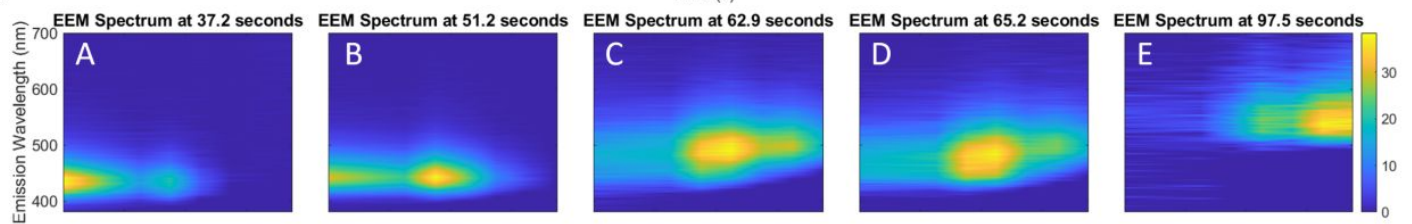

(c)
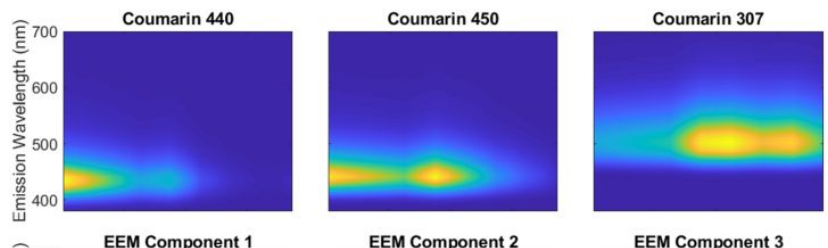

Coumarin 460

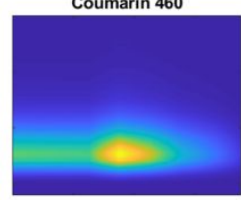

Coumarin $540 \mathrm{~A}$
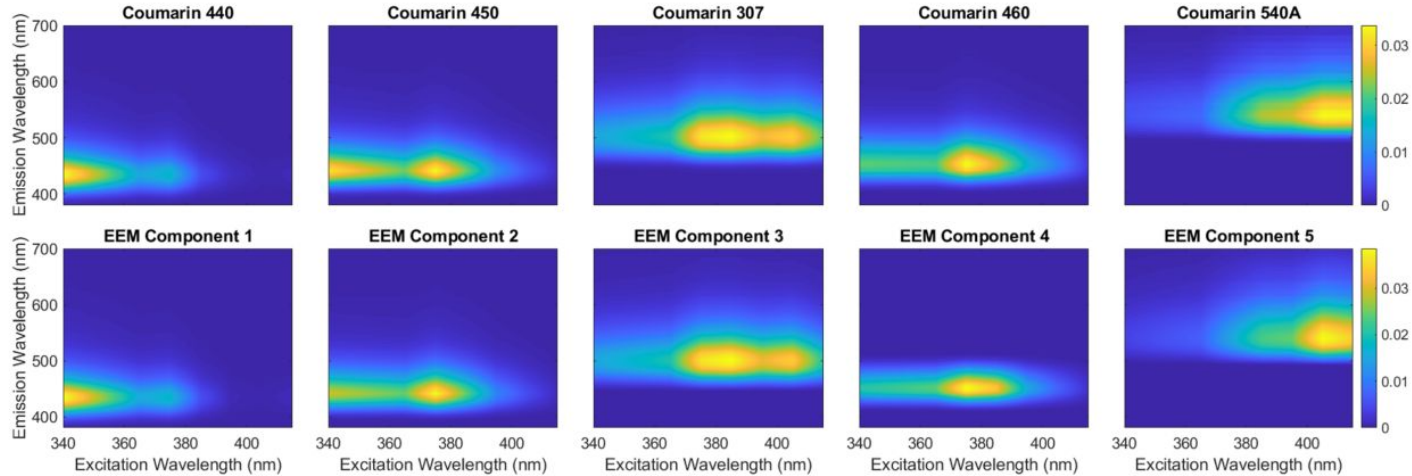

EEM Component 5

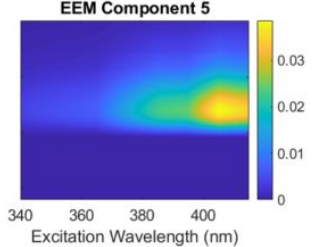

FIGURE S1. Comparison of raw EEM data from HPLC mixture run to the component spectra determined from PARAFAC analysis of single coumarin runs and "blind" PARAFAC model of mixture data.

$$
\% \text { Misattribution }=\frac{\sum_{i}^{\text {misattributed }} \int \boldsymbol{P}_{i}}{\int \boldsymbol{P}_{\boldsymbol{a}}}
$$

The worst case we identified in our study is shown in Figure S2 where part of the signal from peak 1 is attributed to component 2 rather than component 1 . Our experiments on single dye solutions clearly indicated that component 2 does not elute at this time interval. Using the integrated peak areas of the two components we calculated that the signal attributed to component 2 is only $5 \%$ the signal of component 1 .

As stated in the main paper this misattribution is expected to decrease with the addition of more excitation channels.

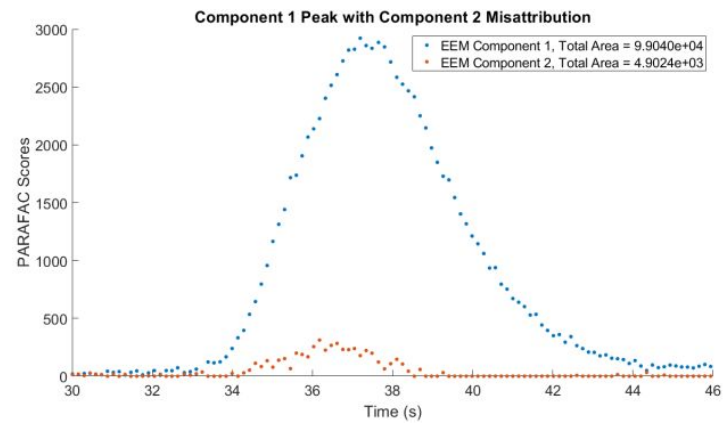

FIGURE S2. Example of misattribution in the form of signal from component 2 attributed to a peak containing only component 1 . The integrated areas of the peaks are listed in the legend.

\section{Component Quantification}

To test the analytical limits of the instrument, serial dilutions of the five coumarin mixture were separated, and the linearity of the EEM measurements and the limits of detection were determined.

Four coumarin mixtures were prepared with each individual coumarin having the same concentration of $50,25,5$ or $1 \mu \mathrm{M}$. These solutions were subjected to HPLC under the same conditions as described in the main paper (fixed $20 \mu \mathrm{L}$ volume injection loop, 4153, Varian ProStar 210, Zorbax SB-C18, 5 $\mu \mathrm{m}, 4.6 \times 150 \mathrm{~mm}$ column, isocratic acetonitrile/water $(80: 20)$ mobile phase at $3.0 \mathrm{~mL} / \mathrm{min}$ ) with a $5 \mathrm{~ms}$ integration time per mask, resulting in a full EEM spectrum acquired every $146 \mathrm{~ms}$.

All five EEM datasets were then combined into a single data cube and processed with PARAFAC modeling for 5 components. The results from that PARAFAC model can be seen in Figure S3. As in the main paper, components 1 through 5 refer to coumarins $440,450,307,460$, and $540 \mathrm{~A}$ respectively. Misattribution is still present within this dataset but interestingly it does appear to scale based on the signal intensity, and low-concentration samples do not display large increases in misattribution. The integrated peak areas were calculated for each component and calibration curves were created to determine the limits of detection for each dye.

The calibration plots seen in Figure S4 do show good linearity supporting the analytical effectiveness of the HT-EEM instrument. The limit of detection (LOD) and limit of quantification (LOQ) were calculated for each of the analytes (Table S1) with an average LOQ and LOD of $10 \mu \mathrm{M}$ and 3 $\mu \mathrm{M}$ respectively. 


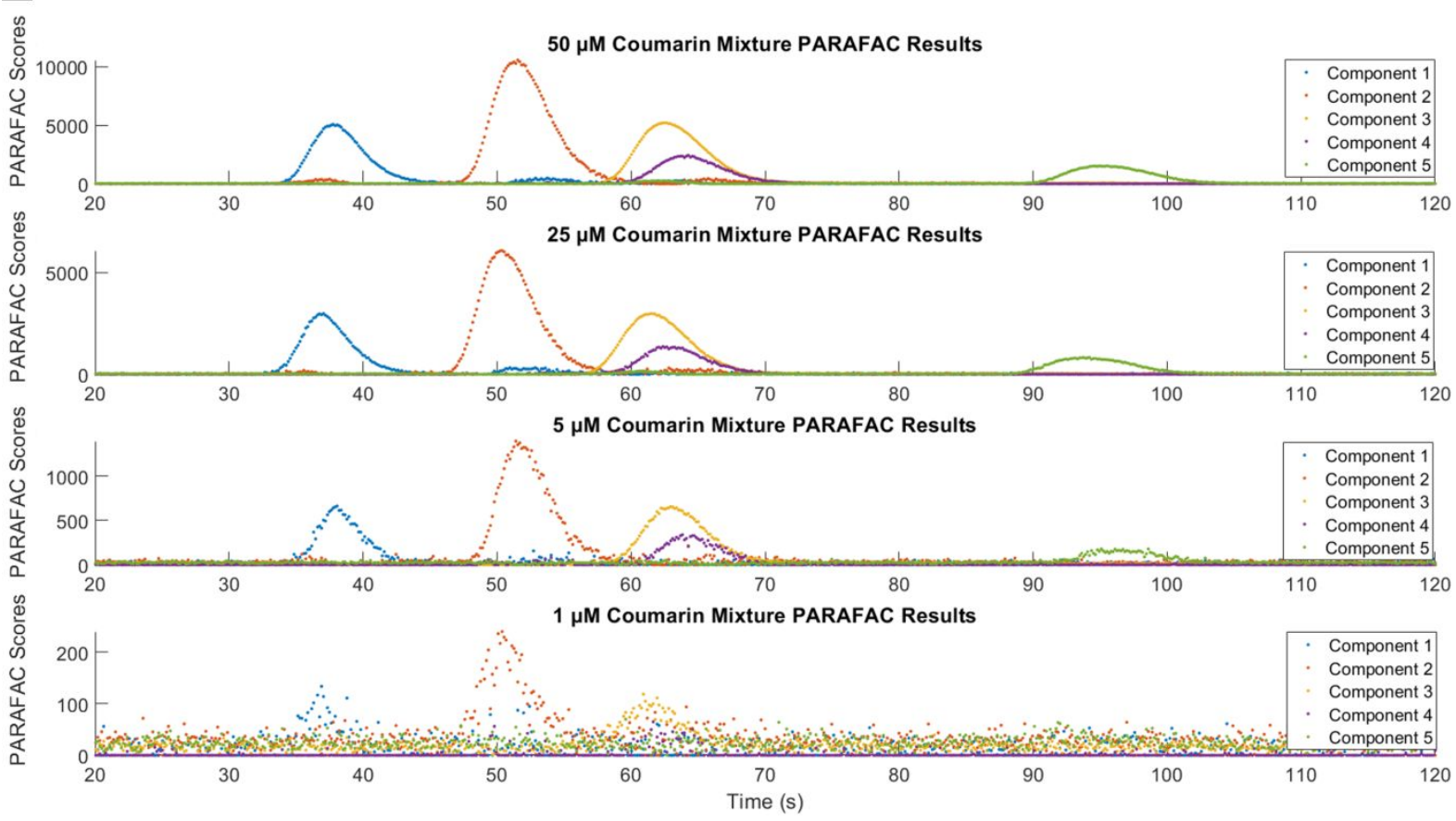

FIGURE S3. PARAFAC scores results from coumarin mixture presented as chromatograms. Components 1 through 5 refer to coumarins $440,450,307,460$, and 540A, respectively.

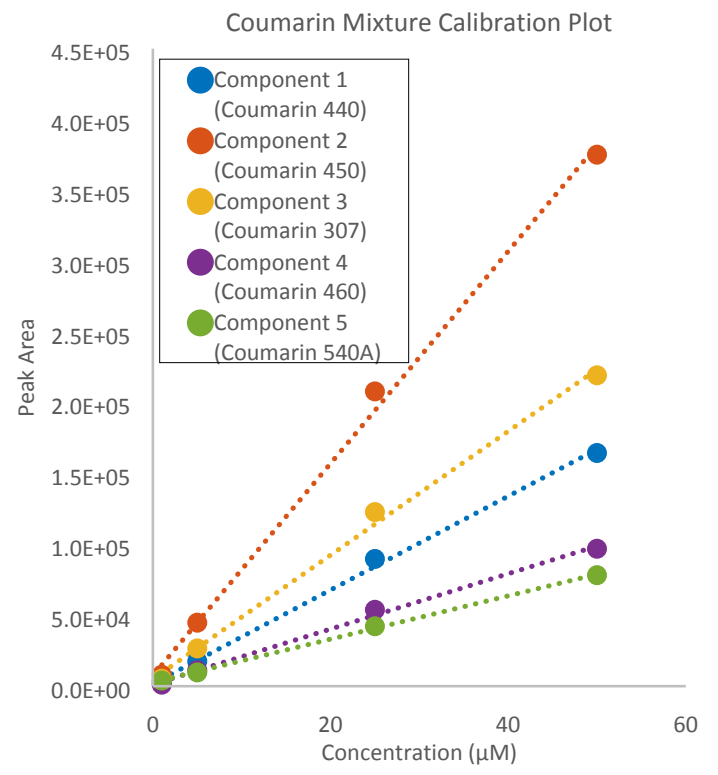

FIGURE S4. Calibration plots for each of the five PARAFAC components. Components 1 through 5 refer to coumarins 440, 450, 307,460 , and 540A respectively.

While important to demonstrate the linearity, sensitivity, and limits of detection of the system, these are highly variable diagnostics which are dependant on many different experimental and physical parameters such as the analyte's quantum yield, EEM integration time, flow rate, and even the mobile phase as polarity affects every fluorophore differently. We expect that the HT-EEM system will work on most mobile phases, gradients, and buffer systems assuming these changes do not cause attenuation of the excitation or emission light, and do not quench the fluorescence of the analyte.

\section{Reference:}

1. Bro, R., Multiway analysis in the food industry. Models, algorithms and applications. Ph.D. dissertation, University of Amsterdam, Amsterdam 2001.

TABLE S1. LOD and LOQ values calculated from the Analysis of Variance of the calibration curves in Fig S4.

\begin{tabular}{r|c|c}
\hline Analyte (Component Number) & LOQ $(\mu \mathrm{M})$ & LOD $(\mu \mathrm{M})$ \\
\hline Coumarin 440 (1) & 10 & 3 \\
\hline Coumarin 450 (2) & 11 & 4 \\
\hline Coumarin 307 (3) & 12 & 4 \\
\hline Coumarin 460 (4) & 13 & 4 \\
\hline Coumarin 540A (5) & 6 & 2 \\
\hline
\end{tabular}

